\title{
A study of nonlinear systems arising in the physics of liquid crystals, using MLPG and DMLPG methods
}

\author{
Ali Shokri ${ }^{1}$ and Erfan Bahmani ${ }^{1}$ \\ ${ }^{1}$ University of Zanjan
}

April 28, 2020

\begin{abstract}
The study of liquid crystals is one of the active areas of research in physics. In this paper, the MLPG and direct MLPG (DMLPG) methods are used for the numerical study of the coupled nonlinear sine-Gordon equations in two dimensions arising from the modeling of some phenomena in the liquid crystals and superconductors. A comparison of numerical results between the MLPG and DMLPG methods highlight the precision and speed of the DMLPG method.
\end{abstract}

\section{Hosted file}

wileyNJD-AMA.pdf available at https://authorea.com/users/306623/articles/437595-a-study-of-nonlinearsystems-arising-in-the-physics-of-liquid-crystals-using-mlpg-and-dmlpg-methods 